\title{
BMJ Open Acupuncture for acute non-specific low back pain: a randomised, controlled, multicentre intervention study in general practice - the Acuback study
}

\author{
Trygve Skonnord (D) , ${ }^{1}$ Holgeir Skjeie (D) ,, 1 Mette Brekke (D) , ${ }^{2}$ Atle Klovning (D) , \\ Margreth Grotle (D) , ${ }^{3,4}$ Eline Aas (D) , ${ }^{5,6}$ Ibrahimu Mdala (D) ,2 Arne Fetveit (D) ${ }^{2}$
}

To cite: Skonnord T, Skjeie H, Brekke M, et al. Acupuncture for acute non-specific low back pain: a randomised, controlled, multicentre intervention study in general practice- - the Acuback study. BMJ Open 2020;10:e034157. doi:10.1136/ bmjopen-2019-034157

- Prepublication history and additional material for this paper are available online. To view these files, please visit the journal online (http://dx.doi org/10.1136/bmjopen-2019034157).

Received 07 September 2019 Revised 02 July 2020 Accepted 03 July 2020

\section{Check for updates}

(c) Author(s) (or their employer(s)) 2020. Re-use permitted under CC BY-NC. No commercial re-use. See rights and permissions. Published by BMJ.

For numbered affiliations see end of article.

\section{Correspondence to}

Trygve Skonnord;

trygve.skonnord@medisin.

uio.no

\section{ABSTRACT}

Objectives The aim of this study was to evaluate whether a single treatment session of acupuncture, when applied in addition to standard treatment for acute low back pain (ALBP), reduces the time to recovery compared with standard treatment alone.

Design A multicentre, randomised, controlled trial. Setting Conducted at 11 Norwegian general practitioners' (GPS') offices.

Participants 171 adults aged $20-55$ years seeking their GP for ALBP ( $\leq 14$ days) between March 2014 and March 2017. Patients with secondary back pain and previous sick leave and acupuncture treatment was excluded.

Interventions The participants were randomised to either the control group (CG) or the acupuncture group (AG) by online software. The $\mathrm{CG}$ received standard treatment according to the Norwegian guidelines, while the AG received one session of Western medical acupuncture treatment in addition to standard treatment. The statistician was blinded to group status.

Primary and secondary outcome measures The primary outcome was median days to recovery. Secondary outcomes were pain intensity, global improvement, backspecific functional status, sick leave, medication and adverse effects.

Results 185 participants were randomised, 95 in the CG and 90 in the AG. 14 participants did not receive the allocated intervention and 4 were excluded from the analysis. Thus, 167 participants were included in the analysis, 86 in the $\mathrm{CG}$ and 81 in the AG. The groups were similar according to baseline characteristics. The median time to recovery was 14 days for the $C G$ and 9 days for the AG, HR 1.37 (95\% Cl 0.95 to 1.96), ( $p=0.089$ ). No serious adverse effects were reported.

Conclusions We did not find any statistically significant reduction in time-to-recovery after a single session of acupuncture for ALBP compared with standard care. Trial registration number NCT01439412.

\section{INTRODUCTION}

Low back pain (LBP) is a common symptom and an important cause of disability globally. ${ }^{12}$ The causes of LBP are multifactorial and most episodes of LBP are categorised as non-specific. ${ }^{13}$ The majority of patients
Strengths and limitations of this study

- The standardised intervention procedures.

- The performance of a pilot study and the development of software led to improved logistics and increased response rate.

- Lower inclusion rates than expected reduced the power, leading to weaker conclusions about the effectiveness of the treatment.

- Trial logistic reasons led to per-protocol analysis instead of intention-to-treat analysis.

affected by acute LBP (ALBP) experiences a decrease in pain and disability within a month, but a significant number will experience recurrences or develop chronic pain. ${ }^{14}$

Most cases of ALBP are treated in primary healthcare. Clinical guidelines for the treatment of ALBP recommend information and education, advice to stay active and to avoid bed rest. ${ }^{5}$ The Norwegian guidelines of 2007 still include pain treatment with paracetamol and/or non-steroidal anti-inflammatory drugs (NSAIDs), ${ }^{6}$ which is nowadays internationally less emphasised ${ }^{57-9}$ In the 2017 US guideline, pharmacological treatment is recommended only if non-pharmacological treatment does not succeed. ${ }^{7}$ Some guidelines recommend acupuncture as first-line treatment, despite lack of high-quality evidence. ${ }^{710}$

In 2013, Lee et al published a systematic review of acupuncture for ALBP and found that evidence is sparse. ${ }^{11}$ They concluded that acupuncture might be more effective than medication for symptom improvement and pain relief than sham acupuncture (SA). However, the authors suggested new trials with better design and reporting of results.

After this systematic review, there have been published four randomised controlled trials (RCTs) of acupuncture for ALBP. ${ }^{12-15}$ Vas et al compared different acupuncture 
types with conventional therapy (CT) and found that the intervention groups fared significantly better than the CT groups. ${ }^{12}$ However, there was no difference between valid acupuncture according to Traditional Chinese Medicine (TCM), SA or placebo acupuncture. Hasegawa et al concluded that Yamanoto's new scalp acupuncture was more effective than sham treatment in ALBP for both pain relief and other outcomes, although their intervention did not reach the predefined values for the primary outcome. ${ }^{14}$ In 2013, Shin et al reported that one session of motion-style acupuncture treatment, consisting of walking with the needles inserted, was superior to one intramuscular injection of diclofenac with respect to pain reduction and function. ${ }^{13}$ In the latest publication for this topic, Fox et al performed a pilot study with 30 participants evaluating a type of ear acupuncture, 'battlefield acupuncture' (BFA). ${ }^{15}$ The authors concluded that BFA was feasible as a non-pharmacological treatment in addition to standard care for LBP in a civilian emergency department. ${ }^{15}$

The idea for the present study was based on clinical experience from general practitioners (GPs), who experienced faster recovery in patients receiving acupuncture for ALBP, often after the first treatment session. We found no other studies with time-to-recovery as the primary outcome, but the single treatment session was supported by two previous studies. ${ }^{131617}$ The treatment was also in accordance with the textbooks on acupuncture. ${ }^{18} 19$

Our study aimed to evaluate whether a single treatment session with acupuncture could result in a faster recovery when applied in addition to standard treatment for ALBP compared with standard treatment alone. Our aim was also to describe pain intensity, disability, work absence, adverse effects and use of medication.

\section{METHODS}

\section{Study design and randomisation}

The study was a multicentre, RCT undertaken in 11 Norwegian GPs' offices. The study period was from March 2014 to March 2017 with the last follow-up in March 2018, after an extension of 1 year due to slow patient recruitment. The participants were randomised by a health secretary into an acupuncture group (AG) or a control group (CG) in a ratio of 1:1, using a web-based randomisation system developed and administered by the Unit of Applied Clinical Research, Norwegian University of Science and Technology, ${ }^{20}$ which performs block randomisation with various block sizes.

Data collection was performed by electronic surveys at 19 different time points; before and after treatment on the day of treatment, and each day for the following 2 weeks; then, after 4 weeks, 12 weeks and 1 year. To administer the logistics of the surveys, we developed software, SESAMe, which is described in a previous publication. ${ }^{21}$

In a prestudy power calculation, we estimated the sufficient sample size to be 135 in each group. ${ }^{22}$ Each patient was blinded to the group allocation when reporting baseline data, but from the time of consultation neither the patient nor the GP was blinded.

The protocol of the present study was published in 2012 and includes further details. ${ }^{22}$ Prior to the main study, we conducted a pilot study that included eight participants from October 2013 to January 2014. The results from the pilot study led to the web-based version of SESAMe, ${ }^{21}$ an exclusion criterion of previous acupuncture, and advices to the participating GP offices about medication standardisation, study logistics and efforts to minimise differences in placebo effects.

The reporting of the study follows the Consolidated Standards of Reporting Trials statement ${ }^{23}$ and the Standards for Reporting Interventions in Clinical Trials of Acupuncture (STRICTA) recommendations. ${ }^{24}$

\section{Participants and recruitment procedure}

Patients with ALBP lasting 14 days or less who contacted their GP office were asked to participate in the trial. We included adults aged 20-55 years with non-specific ALBP who gave informed consent. Exclusion criteria were nerve root affection, 'red flags', pregnancy, disability pension, sick leave of more than 14 days and acupuncture during the last month.

The inclusion/exclusion process was performed by the health secretary at the GP's office and in an initial online survey with information and the consent. She also administered the emails in SESAMe and asked the patient to answer the baseline survey before the consultation. If the GP revealed any exclusion criteria during the consultation, the patient was excluded. This, as well as the time spent in the consultation, was recorded by the GPs.

At each GP office, one GP was trained in acupuncture and treated the AG and from one to four other GPs treated the CG. All acupuncture GPs were specialists in family medicine, and the mean time of acupuncture experience was 7.4 years (range 1-19 years). Nine of the GPs had at least 320 hours of education in acupuncture.

Most-treating GPs in the CG were experienced specialists in family medicine, but some of them were working in the internship programme; thus, the overall experience of the treating GPs varied more than for the AG.

\section{Study interventions}

Standard treatment (CG) consisted of advice about activity, prescription of analgesic medication (paracetamol and/or ibuprofen) and sick leave, if needed, according to the Norwegian national guidelines. ${ }^{6}$

The AG received the same standard treatment as the CG and, in addition, one session of acupuncture treatment with Western medical acupuncture style. This session consisted of $1 \mathrm{~min}$ with two needles of Seirin type B-8a $0.30 \times 30 \mathrm{~mm}$ in the acupuncture points, Lumbar Pain Points (Yaotongxue/Yaotongdian) on the right hand, stimulated to a powerful needle sensation, called 'de Qi' in TCM. With the needles in the hand, the patient was asked to rise and perform mobilisation movements (slow rotating pelvic movements) for $2 \mathrm{~min}$, 
followed by $5 \mathrm{~min}$ on a bench while the patient received six needles of the SEIRIN type J-8 $0.30 \times 50 \mathrm{~mm}$ in the local points Huatuojiaji ('Jiaji') in the L2-L4 segments, stimulated until needle sensation. The needles remained in place until all the needles were removed after a total treatment time of $8-9 \mathrm{~min}$. The short treatment and the choice of only one session of acupuncture were an attempt to reduce potential attention bias. The details of the procedure and the process of choosing the specific and standardised treatment are briefly described in the published protocol, based on clinical experience, literature and feedback from a medical acupuncture expert group. $^{22}$

Prior to the study, the health secretaries and many GPs (including all acupuncture doctors) were gathered at a workshop to ensure they understood the study logistics, the standard ALBP treatment and the standardisation of the acupuncture treatment. During the trial, we arranged two workshops to remind the GP offices of the need for inclusion and update about the study logistics.

\section{Outcome measurements and data collection}

The primary outcome in the study was days to recovery, defined as the first day the patient scored 0 or 1 on the Numerical Rating Scale (NRS). ${ }^{25} 26$ This definition is in line with the definition of 'sustained recovery' with an NRS of 0 or 1 for 7 consecutive days. ${ }^{26}{ }^{27}$ We defined a minimum of 3-day faster recovery as a clinically relevant difference between the groups, based on clinical experience and previous studies. ${ }^{28} 29$

The secondary outcome measurements were pain intensity, ${ }^{25}$ disability by Roland-Morris Disability Questionnaire (RMDQ) ${ }^{30}$ sick leave, 5-point global improvement (Likert scale), use of medication, new visits at the GP's office, health-related quality of life by the EuroQol 5 dimensions, 3 levels (EQ-5D-3L), using UK tariff for time trade-off $^{31}$ and adverse effects. RMDQ and EQ-5D-3L were collected at baseline, 1, 2, 4, 12 weeks and 1 year, while the other secondary outcomes were collected at all time points. In addition, at baseline, we asked for sociodemographic variables, patient preferences for treatment options, expectations with respect to the effect of acupuncture and psychosocial risk profile according to the Örebro screening form for musculoskeletal pain. ${ }^{32} 33$

We also asked the participants in the 1-year survey about the number of new LBP episodes, work absence and whether they had received any other kind of treatments for LBP in the last 9 months.

\section{Patient and public involvement}

No patients were involved in the planning of the study or in the recruitment and the conduct of the study. The study participants were informed that the results of the study would be presented at the study Facebook page. The burden of the intervention could be reported by the patients through the questionnaires of global improvement and adverse events.

\section{Statistical analysis}

Study sample size was calculated to be 270 participants, with $80 \%$ power to detect a 3 days' difference in median time to recovery with an $\alpha$ level of 0.05 and a true HR of 1.429. This was based on the assumption of a 365-day follow-up period, an accrual period of 0 days and a median survival of 7 days. ${ }^{34}$ The study allowed for a dropout rate of up to $10 \%$.

Details of the protocol for randomisation and allocation procedures were published previously. ${ }^{22}$ Statistical analyses were performed using the programs IBM SPSS Statistics V.25 and StataSE V.15. Data were analysed by a statistician who was blinded to group status, and the results presented in tables and figures were finalised before codes were revealed. The analyses were performed per protocol, analysing just participants not excluded during the allocation, lost to follow-up or excluded from analyses of other reasons (figure 1). Participants who did not receive their allocated intervention for some reasons were excluded from the analyses. The NRS data were transformed to the first day of recovery, independent of any intermittent missing answers. We calculated the difference in days to recovery for the two groups using the log-rank test, and late missing answers were censored, leaving the last-specified value for analysis.

The time to recovery was expressed by the median days to recovery for the two groups, and Cox proportional hazard regression models were used to assess the effect of treatment on pain duration (in days). We checked the Cox proportionality assumption and concluded that our model satisfied the assumption of proportionality. Unfortunately, we were not able to use days of pain duration before inclusion as baseline covariate as described in the protocol because this question seemed to be interpreted differently among the participants, as some thought the question was meant to explore the overall history of back pain, not the acute episode.

Numeric secondary outcomes such as NRS were analysed using linear multilevel models with patient random effects, while binary outcomes such as medication use and work absence were analysed using binary multilevel logistic regression models. With numeric outcomes, mean changes over time in the groups were obtained, while estimates of ORs with their $99 \%$ CIs were obtained for binary outcomes.

For primary outcomes, a $p$ value of $<0.05$ was considered statistically significant. For the secondary outcomes, a p value of $<0.01$ was considered significant, and $99 \%$ CIs were given.

\section{RESULTS}

The study flowchart shows that a total of 185 participants who were randomised into the two groups, 167 were included in the analysis, 86 in the CG and 81 in the AG (figure 1). Recruitment of participants at the $11 \mathrm{GP}$ offices varied considerably, and there were also differences in exclusions at each site (see online supplementary file 1). 


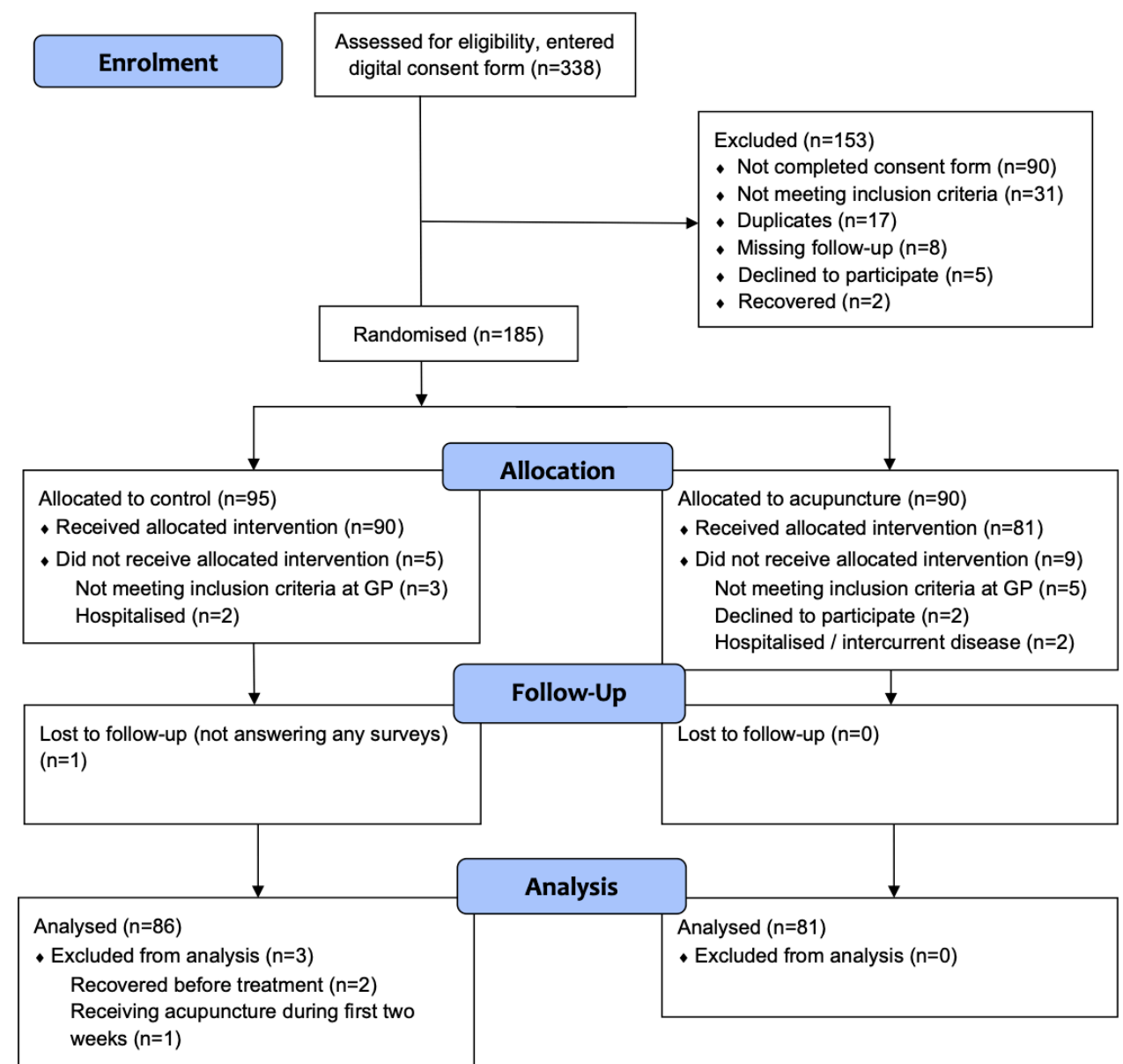

Figure 1 Consolidated Standards of Reporting Trials flow diagram in a trial of acupuncture for acute non-specific low back pain when applied in addition to standard treatment, compared with standard treatment alone.

The overall recruitment was poorer than expected, and even if the inclusion period was extended with 1 year, the planned sample size was not met. Possible causes can be less patients with LBP seeking the GPs due to previous public campaigns, patients seeking other therapists and the circumstances of the trial taking place in busy GP practices with voluntary work by both GPs and health secretaries with no professional research network to help.

The overall response rate in the trial was $87.4 \%$ but varied in each survey and decreased over time. One year into the observation period, 66 participants in the AG and 61 in the CG had answered the survey, resulting in a response rate of $76.0 \%$. Online supplementary file 2 shows the number of missing answers per survey for the primary outcome and online supplementary file 3 for the secondary outcomes. There were no statistically significant differences between the groups in response rate, except for primary outcome at day $2(\mathrm{p}=0.037)$. One participant in the AG underwent an operation for sciatica during the follow-up period.

Table 1 shows the baseline characteristics with sociodemographic data and clinical features of the participants. There were no statistically significant differences between the groups in any of the variables. There were small, non-significantly differences between the groups for obesity and smoking, which are known risk factors to LBP. $^{1}$

The duration of the consultations in the $\mathrm{AG}$ was $20.2 \mathrm{~min}$ (99\% CI 19.0 to 21.5 ) versus $17.0 \mathrm{~min}$ (99\% CI 15.4 to 18.5 ) in the CG, and the difference of $3.2 \mathrm{~min}$ was statistically significant $(\mathrm{p} \leq 0.001)$. In the study, $21.9 \%$ (99\% CI 10.4 to 33.4) of the patients in the CG were treated by their regular GP versus $40.0 \%$ (99\% CI 26.0 to $54.0)$ in the AG $(p=0.011)$.

\section{Primary outcome}

Median time to recovery was 14 days for the CG (IQR 6-84) and 9 days for AG (IQR 4-84). Based on the Cox regression model, the difference of 5 days was not statistically significant, despite achieving the a priori threshold for clinical relevance of 3 days, with a HR 1.37 (95\% CI 0.95 to 1.96$),(\mathrm{p}=0.089)$.

Time to recovery for 365 days and the first 28 days is shown in figure 2 . The log-rank test for 365 days is based on 56 observed and 65.3 expected events in the CG and 64 observed and 54.7 expected events in the AG, which was not statistically significant $(\mathrm{p}=0.072)$. We also performed a sensitivity analysis on the four excluded participants with the same result. Sensitivity analyses with the baseline 
Table 1 Baseline characteristics of participants in a trial of acupuncture for acute non-specific low back pain when applied in addition to standard treatment, compared with standard treatment alone $(n=167)$

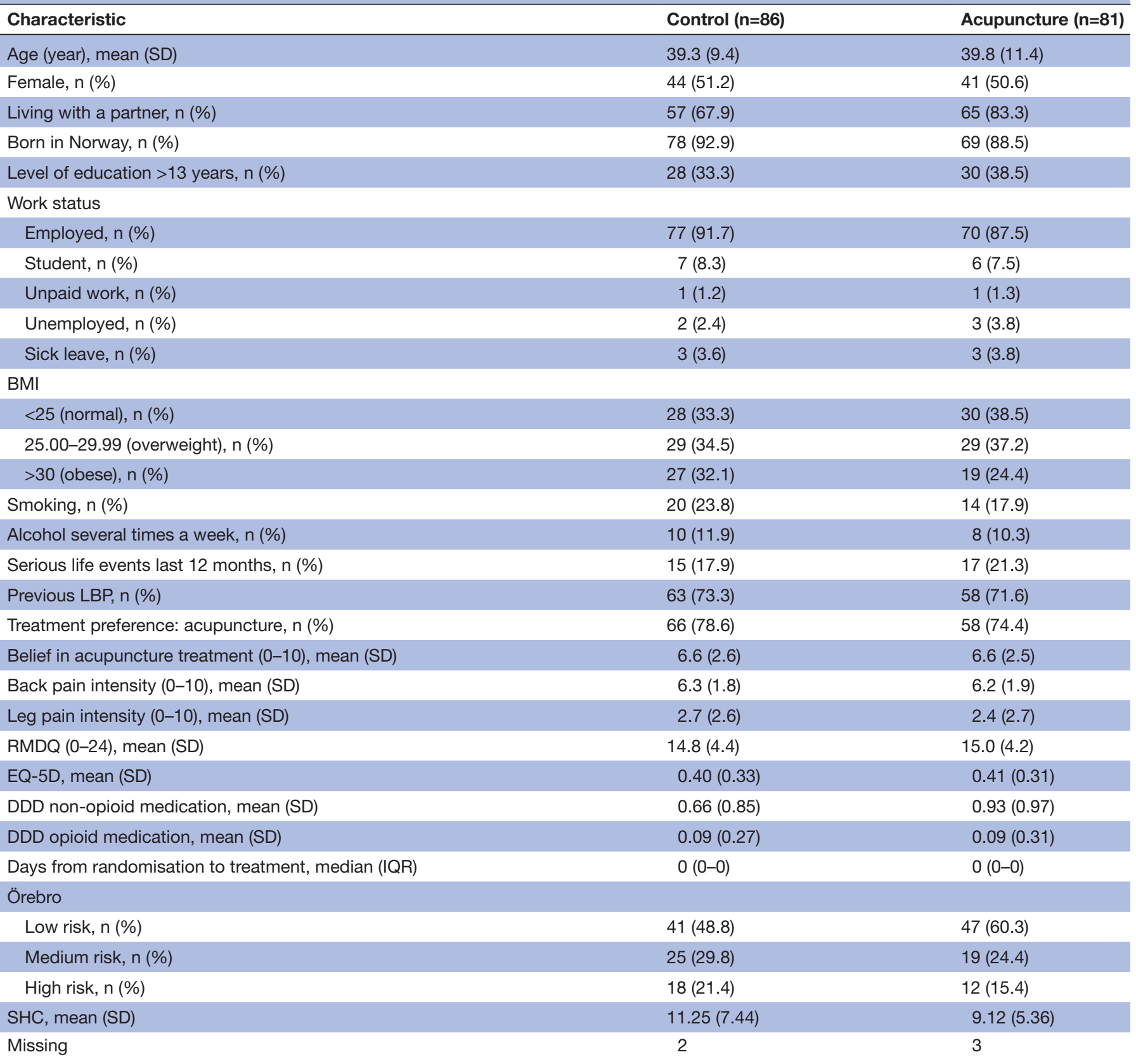

Data in $\mathrm{n}(\%)$, mean (SD) or median (IQR).

There were no significant differences between the groups in any of the variables.

$\mathrm{BMI}$, body mass index; DDD, defined daily dose; EQ-5D, EuroQol, higher score represents better health state; IQR, interquartile range; LBP, low back pain; NRS (0-10), Numerical Rating Scale, higher score represents more pain; RMDQ (0-24), Roland-Morris disability questionnaire, higher score represents greater overall disability; SHC, subjective health complaints, higher score means more reported health complaints.

variables, obesity and smoking, did not change the results of the primary outcome either.

For one extra person to recover during the whole study period, the number needed to treat (NNT) was $7.2(95 \%$ CI 3.7 to 210.3$).{ }^{35}$ This was based on 64 recovered participants in the AG and 56 recovered participants in the CG after 1 year, leading to an absolute risk reduction of 0.139 (95\% CI 0.005 to 0.273$)$.

\section{Secondary outcomes}

Pain intensity during the study period reduced in both groups with no clinically relevant nor statistically significant differences between the two groups at each time point (figure 3). The mean difference in pain between the two groups during the whole study overall was 0.48 $(99 \%$ CI 0.25 to 0.71$)(\mathrm{p}<0.001)$ in favour of the AG. This equals a standardised mean difference (SMD) of 0.13 . 


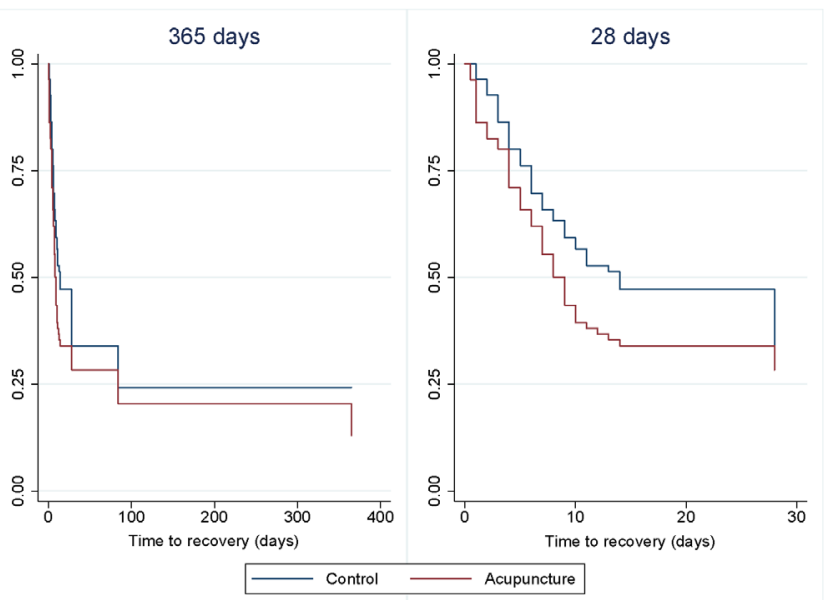

Figure 2 Time to recovery for acute low back pain with acupuncture and standard treatment compared with standard treatment alone. One-year follow-up and first 28 days $(n=167)$.

The same pattern was seen for back-related disability by RMDQ, which showed an improvement during the year for both groups but with no statistically significant difference between the two groups (figure 4).

There were no statistically significant differences in sick leave between the groups at any of the time points (see online supplementary file 4).

The participants' perception of global improvement (feeling better or much better) was highly significantly better in the AG group on day 0 after treatment (OR 8.00, $99 \%$ CI 2.88 to 22.05 ), but later the difference became gradually smaller, with a statistical significance on just one other day (day 4) (see online supplementary file 5).

There were no statistically significant differences in the use of medication, unless for day 3 when fewer participants in the AG used non-opioid medication than in the CG (see online supplementary file 6).

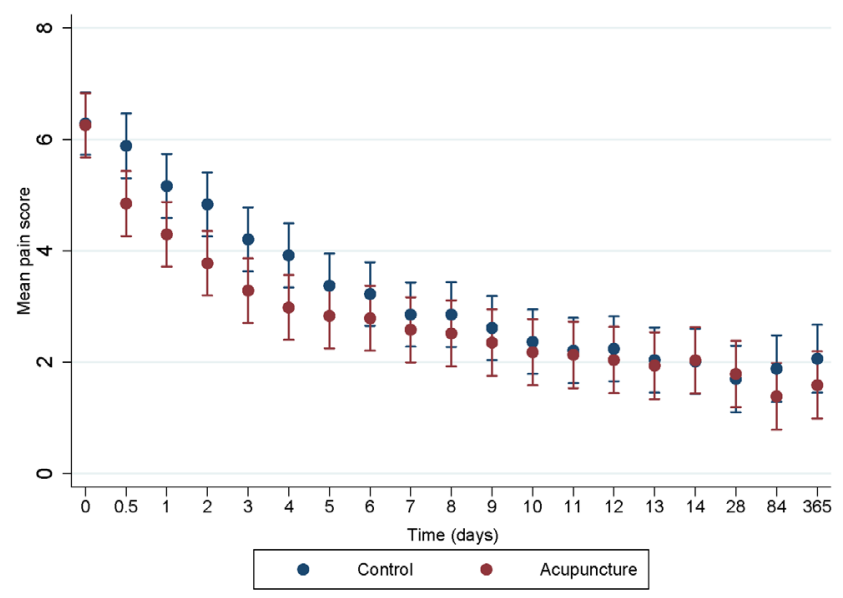

Figure 3 Pain intensity during a 1-year follow-up period in a trial of acupuncture for acute non-specific low back pain when applied in addition to standard treatment, compared with standard treatment alone $(99 \% \mathrm{Cl})$.

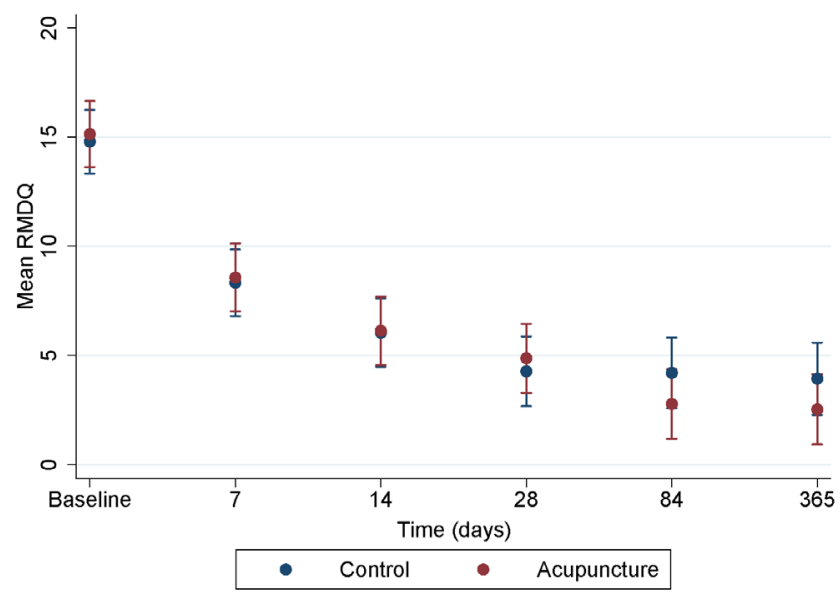

Figure 4 Disability by Roland-Morris Disability Questionnaire (RMDQ) during a 1-year follow-up period in a trial of acupuncture for acute non-specific low back pain when applied in addition to standard treatment, compared with standard treatment alone $(99 \% \mathrm{Cl})$.

The estimated number of new visits to the GP through the study period was 2.7 (99\% CI 2.0 to 3.5 ) in the CG and $2.6(99 \%$ CI 1.9 to 3.3$)$ in the AG, difference $0.1(99 \%$ CI -0.9 to 1.1$)(p=0.76)$. Health-related quality of life measured by EQ-5D-3L did not show statistically significant differences between the two groups at any time point during the study (see online supplementary file 7). There were more, but statistically non-significant, LBP episodes in the CG after 1 year, 3.2 (99\% CI 2.4 to 3.9) versus 2.4 (99\% CI 1.7 to 3.2$)$ in the AG, difference 0.7 (99\% CI -0.3 to 1.8$)(p=0.06)$.

No serious adverse events were reported in the study. Sixteen participants $(18.6 \%, 99 \%$ CI 7.8 to 29.4$)$ in the CG reported some adverse effects compared with 11 (13.6\%, 99\% CI 3.8 to 23.4) in the AG, difference $5.0 \%$ (99\% CI -9.9 to 19.9$)(\mathrm{p}=0.38)$. Two participants reported pain/ soreness in their hand the day after the treatment because of the needles. Twenty-two participants reported gastrointestinal symptoms, 14 of them in the CG. Other less frequent symptoms were tiredness, headache, dyspnoea and muscle pain.

\section{DISCUSSION \\ Principle findings}

This study showed that adding one single session of 8-9 min of acupuncture treatment to standard guidelinebased care to patients with ALBP resulted in 5 days faster recovery of pain, but the result was not statistically significant. Similarly, adding acupuncture to standard guideline-based primary care did not show any statistically significant effect in the secondary outcome measures of disability, work absence and quality of life. For the secondary outcomes of pain, self-reported global improvement and medication, we found small differences without clinical relevance. Finally, the acupuncture 
treatment was safe, with no significant differences of major symptoms or serious adverse events.

\section{Strengths and limitations of the study}

The main strength of this study was the standardised intervention procedures, leading to no attention bias between the two groups. Another strength was the performance of a pilot study that led to logistic changes that contributed to both an equality of the groups and an improved response rate. The innovative process of developing our own logistic software (SESAMe) was central in this quality improvement. ${ }^{21}$

The main limitation of this study was the low power due to lower inclusion rates than expected, even after we extended the inclusion period with 1 year. This led to weaker conclusions about the effectiveness of the treatment. The results of the primary outcome could well be due to a type II error. However, low power in a trial reduces the likelihood that the observed effect represents a true effect. ${ }^{36}$ The wider SDs in an underpowered study make it more likely to reach clinical relevant values. ${ }^{36}$ The very small effect size on pain $(\mathrm{SMD}=0.13)$ and the lack of effect on disability can imply that the 5 days faster time to recovery can be a spurious finding. Another limitation is that we were not able to perform the intended intentionto-treat analysis. Of logistic reasons, we had to perform the last eligibility evaluation by the GP in the consultation. That is why 14 participants were randomised but excluded before intervention was given. In addition, four participants were excluded from analysis, three of them because of statistical challenges (left censoring) and one because of exclusion criteria. However, a sensitivity analysis did not change the results. On the other hand, the exclusion after randomisation may have caused bias. Lack of fidelity checklist to measure the fidelity of the interventions is another limitation. Even considering the significance level of 0.01 on secondary outcomes, with the large number of statistical tests performed, there is a possibility that any of the observed differences could be due to false positives. In addition, many of the CIs are wide, so the estimated effects lack precision.

\section{Relation to other studies}

The acupuncture treatment provided in this trial consisted of both shorter treatment time and fewer treatment sessions than usual. ${ }^{37} 38$ This may have caused less chances to detect a real difference in effectiveness. On the other side, a longer treatment time and more sessions could have caused more attention bias. Our results could not support Vas et al showing the effectiveness of acupuncture compared with CT. ${ }^{12}$ The short-term effect of only one acupuncture treatment session for LBP was previously shown by Shin $e t a l^{13}$ but MacPherson et al showed that pain outcomes were influenced by increased numbers of needles and more sessions, and thus the dose was important. ${ }^{38}$ After the trials of Vickers and MacPherson, ${ }^{38} 39$ the US National Center for Complementary and Integrative Health (NCCIH) announced a need for pragmatic acupuncture trials for pain management, testing the effectiveness in 'real-world' conditions, while efficacy studies seek effect under ideal conditions. ${ }^{40} 41$ Because this was a pragmatic trial in accordance with the NCCIH recommendations, the participants and GPs were not blinded. Some may argue that this is a problem in acupuncture trials, and it would be a limitation in an efficacy study. However, a large systematic review with individual patient data meta-analysis by Vickers $e t$ al in 2012 has evaluated the efficacy of acupuncture for pain, and the authors showed that acupuncture has a small, specific effect on pain. ${ }^{39}$ The difference between true acupuncture and sham or placebo acupuncture is small, and trials will need large sample sizes to emphasise these differences, which Vas et aldemonstrated to be also true for ALBP. ${ }^{12}$ In our study, there were non-significant differences in pain for each time point, but statistically significant difference in pain overall. Because the effect size was very small and the difference was considered not clinically relevant, this result should be interpreted with caution.

The highly significant difference in the early perception of global improvement could be a result of the positive expectations, but it could also be due to the experience of a faster recovery with less pain. The findings are in accordance with the systematic review by Lee $e t$ al in which acupuncture is compared with the use of NSAIDs. ${ }^{11}$ However, subjective outcomes have been shown to exaggerate effect estimates in trials that were not blinded. ${ }^{42} \mathrm{In}$ addition, the slightly higher response rates in the AG the first days could have contributed to a possible strengthening of the positive subjective outcomes.

The two study groups scored equally for treatment preferences and belief in acupuncture prior to the treatment. For the AG, this might represent a positive expectation bias when receiving the treatment, while those in the CG might have had a negative expectation bias when not receiving the acupuncture they had wanted. This would be in accordance with other research demonstrating an effect of treatment preferences and belief in the treatment in pain studies. ${ }^{43}$

There are not many trials of non-pharmacological treatments reporting NNT. Despite the lack of effect between the two groups in the present study, the NNT from our trial was comparable to both other LBP trials ${ }^{45} 46$ and acupuncture trials. ${ }^{478}$

The few observed differences between the two groups can be due to specific and non-specific needle effects, the contribution of the mobilisation movements, the extra consultation time or the attention bias provided by the overall extra treatment ritual. There could also be an operator effect of a less or more enthusiastic behaviour in the consultation. The patient-practitioner relationship is shown to influence the placebo effect, even in standardised intervention procedures. ${ }^{49}$ However, this could be a phenomenon in both groups, and also influenced by the prescribing of medication, performing a physical examination or not, empathic behaviour and time spent. Short consultation times are a key challenge 
to implementing best practices for $\mathrm{LBP}^{5}$ but in our study, we cannot conclude whether the extra time for acupuncture compensated for possibly less time for giving advice.

More participants in the AG than in the CG met with their regular GP during the consultation. Continuity in the doctor-patient relationship, including previous knowledge about the patient, is associated with improved patient outcomes. ${ }^{5051}$

\section{CONCLUSION}

This trial showed that adding one treatment session of acupuncture in combination with mobilisation movements had similar effect as usual care for patients with ALBP during 1 year of follow-up. On primary outcome, the observed difference of 5 days earlier recovery in the AG was not statistically significant. On secondary outcomes, there were no statistically significant differences in self-reported outcome measures of disability and health-related quality-of-life. On pain reduction, there was a statistically significant but not clinically relevant difference.

\section{Author affiliations}

${ }^{1}$ Department of General Practice, Institute of Health and Society, University of Oslo, Oslo, Norway

${ }^{2}$ General Practice Research Unit (AFE), Department of General Practice, Institute of Health and Society, University of Oslo, Oslo, Norway

${ }^{3}$ Department of Physiotherapy, Oslo Metropolitan University, Oslo, Norway

${ }^{4}$ Research and Communication Unit for Musculoskeletal Health, Oslo

universitetssykehus Ulleval, Oslo, Norway

${ }^{5}$ Department of Health Management and Health Economics, Institute of Health and Society, University of Oslo, Oslo, Norway

${ }^{6}$ Health Services Research Unit, Akershus University Hospital, Lorenskog, Norway

\section{Twitter Trygve Skonnord @trygvets}

Acknowledgements The authors would like to thank the participating GPs and health secretaries, and Finn Steen whose contribution to the data collection was essential through the development of SESAMe.

Contributors TS and HS had the idea for the project. TS, HS, MB, MG and AF contributed to conceptualisation and design of the study. TS and AK developed the software for data collection. TS and IM performed the statistical analyses. TS and EA performed the health-economic analyses. TS drafted the article. All authors have discussed the results and revised this manuscript critically for important intellectual content. All authors read and approved the final manuscript.

Funding This study was funded by Institute of Health and Society, University of Oslo. The study received also some funding for logistic expenses by private endowments, the 'Gidske and Peter Jacob Sørensens fund' and the 'Trygve Gythfeldts fund'.

Competing interests None declared.

Patient consent for publication Not required.

Ethics approval Ethical approval was given by the Regional Ethics Committee of South-Eastern Norway (reference 2013/611/REK sør-øst A).

Provenance and peer review Not commissioned; externally peer reviewed.

Data availability statement Data are available upon reasonable request. The additional unpublished data are available from the corresponding author on request.

Open access This is an open access article distributed in accordance with the Creative Commons Attribution Non Commercial (CC BY-NC 4.0) license, which permits others to distribute, remix, adapt, build upon this work non-commercially, and license their derivative works on different terms, provided the original work is properly cited, appropriate credit is given, any changes made indicated, and the use is non-commercial. See: http://creativecommons.org/licenses/by-nc/4.0/.

\section{ORCID iDs}

Trygve Skonnord http://orcid.org/0000-0003-4816-076X Holgeir Skjeie http://orcid.org/0000-0002-7586-8651

Mette Brekke http://orcid.org/0000-0003-3454-2329 Atle Klovning http://orcid.org/0000-0002-2917-1845

Margreth Grotle http://orcid.org/0000-0001-8243-1143

Eline Aas http://orcid.org/0000-0002-9878-6165

Ibrahimu Mdala http://orcid.org/0000-0002-5204-1934

Arne Fetveit http://orcid.org/0000-0001-8566-1972

\section{REFERENCES}

1 Hartvigsen J, Hancock MJ, Kongsted A, et al. What low back pain is and why we need to pay attention. Lancet 2018;391:2356-67.

2 GBD 2015 Disease and Injury Incidence and Prevalence Collaborators. Global, regional, and national incidence, prevalence, and years lived with disability for 310 diseases and injuries, 19902015: a systematic analysis for the global burden of disease study 2015. Lancet 2016;388:1545-602.

3 Maher C, Underwood M, Buchbinder R. Non-specific low back pain. Lancet 2017;389:736-47.

4 Vasseljen O, Woodhouse A, Bjørngaard $\mathrm{JH}$, et al. Natural course of acute neck and low back pain in the general population: the HUNT study. Pain 2013;154:1237-44.

5 Foster NE, Anema JR, Cherkin D, et al. Prevention and treatment of low back pain: evidence, challenges, and promising directions. Lancet 2018;391:2368-83.

6 Laerum E, Brox JI, Storheim K, et al. National clinical guidelines. Iow back pain with and without nerve root affection. Oslo: Formi, 2007.

7 Qaseem A, Wilt TJ, McLean RM, et al. Noninvasive treatments for acute, subacute, and chronic low back pain: a clinical practice guideline from the American College of physicians. Ann Intern Med 2017;166:514-30.

8 Stochkendahl MJ, Kjaer P, Hartvigsen J, et al. National clinical guidelines for non-surgical treatment of patients with recent onset low back pain or lumbar radiculopathy. Eur Spine J 2018;27:60-75.

9 Bernstein IA, Malik Q, Carville S, et al. Low back pain and sciatica: summary of NICE guidance. BMJ 2017;356:i6748.

10 Oliveira CB, Maher CG, Pinto RZ, et al. Clinical practice guidelines for the management of non-specific low back pain in primary care: an updated overview. Eur Spine J 2018;27:2791-803.

11 Lee J-H, Choi T-Y, Lee MS, et al. Acupuncture for acute low back pain: a systematic review. Clin J Pain 2013;29:172-85.

12 Vas J, Aranda JM, Modesto M, et al. Acupuncture in patients with acute low back pain: a multicentre randomised controlled clinical trial. Pain 2012;153:1883-9.

13 Shin J-S, Ha I-H, Lee J, et al. Effects of motion style acupuncture treatment in acute low back pain patients with severe disability: a multicenter, randomized, controlled, comparative effectiveness trial. Pain 2013;154:1030-7.

14 Hasegawa TM, Baptista AS, de Souza MC, et al. Acupuncture for acute non-specific low back pain: a randomised, controlled, doubleblind, placebo trial. Acupunct Med 2014;32:109-15.

15 Fox LM, Murakami M, Danesh $\mathrm{H}$, et al. Battlefield acupuncture to treat low back pain in the emergency department. Am J Emerg Med 2018;36:1045-8.

16 Shin J-S, Ha I-H, Lee T-G, et al. Motion style acupuncture treatment (MSAT) for acute low back pain with severe disability: a multicenter, randomized, controlled trial protocol. BMC Complement Altern Med 2011;11:127.

17 Araki S, Kawamura O, Mataka T, et al. Randomized controlled trial comparing the effect of manual acupuncture with sham acupuncture for acute low back pain. J Japan Soc Acupun Moxibus 2001;51:382.

18 Deadman P, Al-Khafaji M, Baker K. A manual of acupuncture. J Chinese Med 2008:574-80.

19 Heyerdahl O, Lystad N. Textbook of acupuncture. Oslo: Universitetsforlaget, 2007: 291-303.

20 Norwegian University of Science and Technology. Unit of applied clinical research 2019. Available: https://www.ntnu.edu/mh/akf and http://www.webcitation.org/78NoisCOH

21 Skonnord T, Steen F, Skjeie H, et al. Survey email scheduling and monitoring in eRCTs (SESAMe): a digital tool to improve data collection in randomized controlled clinical trials. J Med Internet Res 2016;18:e311.

22 Skonnord T, Skjeie H, Brekke M, et al. Acupuncture for acute nonspecific low back pain: a protocol for a randomised, controlled multicentre intervention study in general practice-the acuback study. BMJ Open 2012;2:e001164. 
23 Schulz KF, Altman DG, Moher D, et al. Consort 2010 statement: updated guidelines for reporting parallel group randomised trials. BMJ 2010;340:c332.

24 MacPherson H, Altman DG, Hammerschlag R, et al. Revised standards for reporting interventions in clinical trials of acupuncture (stricta): extending the CONSORT statement. Acupunct Med 2010;28:83-93.

25 Grotle M, Brox JI, Vøllestad NK. Concurrent comparison of responsiveness in pain and functional status measurements used for patients with low back pain. Spine 2004;29:E492-501.

26 Chiarotto A, Boers M, Deyo RA, et al. Core outcome measurement instruments for clinical trials in nonspecific low back pain. Pain 2018:159:481-95

27 Williams CM, Maher CG, Latimer J, et al. Efficacy of paracetamol for acute low-back pain: a double-blind, randomised controlled trial. Lancet 2014;384:1586-96.

28 Hancock MJ, Maher CG, Latimer J, et al. Assessment of diclofenac or spinal manipulative therapy, or both, in addition to recommended first-line treatment for acute low back pain: a randomised controlled trial. Lancet 2007;370:1638-43.

29 Williams CM, Latimer J, Maher CG, et al. PACE-the first placebo controlled trial of paracetamol for acute low back pain: design of a randomised controlled trial. BMC Musculoskelet Disord 2010;11:169.

30 Roland M, Morris R. A study of the natural history of back pain. Part I: development of a reliable and sensitive measure of disability in lowback pain. Spine 1983;8:141-4.

31 Solberg TK, Olsen J-A, Ingebrigtsen T, et al. Health-related quality of life assessment by the EuroQol-5D can provide cost-utility data in the field of low-back surgery. Eur Spine J 2005;14:1000-7.

32 Linton SJ, Halldén K. Can we screen for problematic back pain? a screening questionnaire for predicting outcome in acute and subacute back pain. Clin J Pain 1998;14:209-15.

33 Grotle M, Vøllestad NK, Brox Jl. Screening for yellow flags in firsttime acute low back pain: reliability and validity of a Norwegian version of the acute low back pain screening questionnaire. Clin J Pain 2006;22:458-67.

34 Schoenfeld D. Find statistical considerations for a study where the outcome is a time to failure 2001. Available: http://hedwig.mgh. harvard.edu/sample size/time to event/para time.html

35 Altman DG. Confidence intervals for the number needed to treat. BMJ 1998;317:1309-12.

36 Button KS, loannidis JPA, Mokrysz C, et al. Power failure: why small sample size undermines the reliability of neuroscience. Nat Rev Neurosci 2013;14:365-76.

37 Heyerdahl O, Lystad N. Treatment strategy: textbook of acupuncture (p: 233-34, 313-14). Oslo, Norway: Universitetsforlaget, 2013.
38 MacPherson $\mathrm{H}$, Maschino AC, Lewith G, et al. Characteristics of acupuncture treatment associated with outcome: an individual patient meta-analysis of 17,922 patients with chronic pain in randomised controlled trials. PLoS One 2013;8:e77438.

39 Vickers AJ, Cronin AM, Maschino AC, et al. Acupuncture for chronic pain: individual patient data meta-analysis. Arch Intern Med 2012;172:1444-53.

40 The National Center for Complementary and Integrative Health $(\mathrm{NCClH})$. Acupuncture research - areas of high and low programmatic priorities 2015. Available: https://nccih.nih.gov/grants/ acupuncture/priorities

41 Witt CM. Efficacy, effectiveness, pragmatic trials-guidance on terminology and the advantages of pragmatic trials. Forsch Komplementmed 2009;16:292-4.

42 Wood L, Egger M, Gluud LL, et al. Empirical evidence of bias in treatment effect estimates in controlled trials with different interventions and outcomes: meta-epidemiological study. BMJ 2008;336:601-5.

43 Pariente J, White P, Frackowiak RSJ, et al. Expectancy and belief modulate the neuronal substrates of pain treated by acupuncture. Neuroimage 2005;25:1161-7.

44 Linde K, Witt CM, Streng A, et al. The impact of patient expectations on outcomes in four randomized controlled trials of acupuncture in patients with chronic pain. Pain 2007;128:264-71.

45 Moore RA, Smugar SS, Wang H, et al. Numbers-needed-to-treat analyses-do timing, dropouts, and outcome matter? Pooled analysis of two randomized, placebo-controlled chronic low back pain trials. Pain 2010;151:592-7.

46 Parkin-Smith GF, Norman IJ, Briggs E, et al. A structured protocol of evidence-based conservative care compared with usual care for acute nonspecific low back pain: a randomized clinical trial. Arch Phys Med Rehabil 2012;93:11-20.

47 Linde K, Allais G, Brinkhaus B, et al. Acupuncture for the prevention of episodic migraine. Cochrane Database Syst Rev 2016;6:CD001218.

48 Jensen R, Gøthesen O, Liseth K, et al. Acupuncture treatment of patellofemoral pain syndrome. J Altern Complement Med 1999:5:521-7.

49 Kelley JM, Lembo AJ, Ablon JS, et al. Patient and practitioner influences on the placebo effect in irritable bowel syndrome. Psychosom Med 2009;71:789-97.

50 Tammes P, Salisbury C. Continuity of primary care matters and should be protected. BMJ 2017;356:j373.

51 Hjortdahl P. Continuity of care: general practitioners' knowledge about, and sense of responsibility toward their patients. Fam Pract 1992;9:3-8. 